Journal of Systems Science and Information

Feb., 2015, Vol. 3, No. 1, pp. 59-67

DOI: $10.1515 /$ jssi-2015-0059

\title{
Designing the Optimal Extended Warranty Price with Indirect Network Effect
}

\author{
Na TAO \\ School of Management, Xi'an Jiaotong University, Xi'an 710049, China \\ E-mail: taonana2000@126.com \\ Sheng ZHANG \\ School of Public Policy and Administration, Xi'an Jiaotong University, Xi'an 710049, China \\ E-mail: zhangsheng@xjtu.edu.cn
}

\begin{abstract}
In the situation of indirect network effect, this paper mainly studies the optimal extended warranty price. First, the characteristics of the extended warranty price are discussed. Next, the optimal extended warranty price model is advanced. From the model, the authors can draw that the influence factors mainly include the producer and consumers' risk preferences, the incompatible degree of the producer's maintenance technology, the extended warranty period, the producer and consumers' per-occasion maintenance cost. Finally, the authors carry the simulation method to indicate their influence relation. The results have theoretical significance for the automobile producer to ensure the optimal extended warranty price.
\end{abstract}

Keywords extended warranty price; maintenance cost; risk preference; failure rate

\section{Introduction}

The utility that the consumers derive from the durable product mainly depends on the availability of complementary product (or services). The more available the complementary product (or services) are, the more purchase intention consumers have. The effect is known as indirect network effect. Katz and Shapiro ${ }^{[1]}$ defined the product with indirect network effect as system product which are composed of hardware product and software product. The demand for the hardware product is indirectly impacted by the increased supply of complementary software product.

The domestic automobile can't be persistently developed by introducing the technology during the short period as the high quality of the imported automobile seriously threatens the development of the domestic automobile. The paper avoids the disadvantaged of domestic automobile development and proposes the system competing strategy that is improving hardware technology and software quality. The paper considers the optimal extended warranty price with indirect network effect. The paper analyzes the mechanism of indirect network effect and market competition strategy which provides theory support for the extended warranty. The optimal extended warranty price is analyzed. Based on the positive feedback mechanism of

Received January 22, 2014, accepted September 1, 2014

Supported by the Soft Science Major Projects of Shaanxi Province of China (2013KRZ01) 
indirect network effect, we draw the following conclusion that the variety of the software product will expand the installation base of hardware product. For automobile producer, they will increase the maintenance institution. The effective way to increase the producer's profit is that the producer should enhance the intensity of the indirect network effect and improve consumers' switch cost. For automobile producer, they will provide the extended warranty when the basic warranty expires to continually lock consumers.

Berke and Zaino ${ }^{[2]}$ pointed that warranty are the strategy to expand market share for the producer. The warranty is viewed as a selling argument. The customers will view warranty as compensation from the producer, which insures that the defective entities will be repaired or replaced during the warranty period at no cost or at a low cost. Lutz and Padmanabhan ${ }^{[3]}$ found that the extended warranty demand is larger when the consumes' risk preference is aversion and the basic warranty period is short. Chun and Tang ${ }^{[4]}$ studied the influence factors of warranty price that is the producer and consumers' anticipate failure rate. Padmanabhan ${ }^{[5]}$ proposed the opportunity cost awaiting maintenance for high income people is high. Spence ${ }^{[6]}$ suggested that the higher the warranty degree is, the higher the product quality is. Lutz ${ }^{[7]}$ discussed the method of warranty transmits the product' quality in consumers' information asymmetry. Heal ${ }^{[8]}$ put forward that warranty transfers the consumers' risk for uncertainty failure. Akerlof ${ }^{[9]}$ pointed that the poor quality product drive out the good quality product as the uncertainty product quality.

Padmanabhan and Rao ${ }^{[10]}$ constructed the mathematical model to show that the producer may combine the basic warranty and extended warranty to satisfy the customers' demand. Chen and Ross ${ }^{[1]}$ regarded warranties can influence the perception and purchase desire of product for consumers. Ladany and Shore ${ }^{[12]}$, Chen and Chien ${ }^{[13]}$ proposed warranty is the signal for the producer to transmit the product' quality. Murthy and Blischke ${ }^{[14]}$, Price and Dawar $^{[15]}$ emphasized that the producer should consider the following relation: Design quality, product quality and service quality when warranty is designed. Lawless et al. ${ }^{[16]}$ considered the warranty is the effective way to transmit the product quality. Zhou et al. ${ }^{[17]}$ built the model to detect the product quality of warranty data base. $\mathrm{Wu}^{[18]}$ thought the warranties are not only is reduced by the failure, but also is triggered as consumers' habit. Lawless et al. ${ }^{[19]}$ deemed that the two factors inducing the product failure are the product life and the clients' habit. Ye et al. ${ }^{[20]}$ recognized consumers' good use habit positively influences the product life proposing the failure model.

The rest of this paper is structured as follows. In Section 2, the paper studies the feature of the extended warranty. The optimal extended warranty price is obtained in Section 3. The impact factors on the extended warranty are analyzed by simulation in Section 4. Conclusions are presented in the concluding section.

\section{The Mathematic Model}

\subsection{The Feature of the Extended Warranty Price}

Suppose the number of consumers is $n_{1}$. Assuming the consumers have real purchase. Each consumer purchases one item. So the total number of products sold is $n_{1}$. Suppose that the proportion of items sold with warranty is $\sigma$ so that $\sigma n_{1}$ items are sold with warranty 
and $(1-\sigma) n_{1}$ items without warranty. The product failure rate is different in the operation. Let $\tau_{w}$ be the total number of product failures of the $\sigma n$ warranted items; Let $\tau_{n}$ denote the total number of product failures of the $(1-\sigma) n_{1}$ non-warranted items. The producer and consumers' per-occasion maintenance cost are $r_{p}$ and $r_{c}$. When the consumers purchase the extended warranty, the $\sigma n_{1}$ items are maintenance free of charge during the extended warranty period. The consumers who don't buy the extended warranty must pay the full maintenance cost. In the situation, the consumers will have two choices: The original producer or the other producer provides the maintenance service. Let $h$ denote the proportion of non-warranted items that will be repaired by the original producer.

The cost for the consumers with warranty is the extended warranty price $C_{E} \sigma n_{1}$. The cost for the consumers non-warranty is the maintenance cost which equals to the per-occasion maintenance cost multiply by the total number of product failures $\tau_{n} r_{c}$. If $C_{E} \sigma n_{1}<\tau_{n} r_{c}$, the consumers will choose to purchase the extended warranty. If $C_{E} \sigma n_{1}>\tau_{n} r_{c}$, the consumers won't choose to purchase the extended warranty.

The extended price model in the paper is based on several assumptions. The first assumption is that the product failure rate is constant. As per-occasion maintenance cost burden by the consumers mainly includes service cost and replacement cost which are fixed. The factor determined the consumers' purchase desire is the total number of product failures of the $(1-\sigma) n_{1}$ non-warranted items. The total number of product failures during the extended warranty period is distributed as Gamma distribution $\psi_{c} \sim g_{c}\left(\nu_{c} \mid \alpha_{c}, \beta_{c}\right) . \alpha_{c}$ and $\beta_{c}$ are the shape parameter and scale parameter.

The second assumption is on the producer and consumers' utility function. As most consumers prefer lower cost to higher cost, the customers' utility function $U_{c}$ is concave and strictly decreasing. We have $U_{c}^{\prime}<0, U_{c}^{\prime \prime}<0$. The concave function implies that the consumers' risk preference is risk aversion. As most producers prefer higher profit to lower profit, the producer's utility function $U_{p}$ is concave and strictly increasing. We have $U_{p}^{\prime}>0, U_{p}^{\prime \prime}<0$. The concave function implies that the producer's risk preference is risk aversion.

When the product failure rate complies with Equation (1), the customers' utility function of purchasing the extended warranty and non-warranted is indifference.

$$
E\left[U_{c}\left(C_{E} \sigma n_{1}\right)\right]=E\left[U_{c}\left(\tau_{n} r_{c}\right)\right]=\sum_{\tau_{n}=0}^{\infty} U_{c}\left(\tau_{n} r_{c}\right) p\left(\tau_{n} \mid \psi_{c}=\nu_{c}^{*}\right)
$$

If $\psi_{c}>\nu_{c}{ }^{*}, E\left[U_{c}\left(C_{E} \sigma n_{1}\right)\right]>E\left[U_{c}\left(\tau_{n} r_{c}\right)\right]$, the utility of purchasing the extended warranty is more than the utility non-warranted items. So the consumers will choose to buy the extended warranty. The proportion of items sold with warranty is

$$
\sigma=\int_{\nu_{c}^{*}}^{\infty} g_{c}\left(\nu_{c} \mid \alpha_{c}, \beta_{c}\right) \mathrm{d} \nu_{c}=1-G_{c}\left(\nu_{c}^{*} \mid \alpha_{c}, \beta_{c}\right)
$$

As we can see from Equation (2), the main factor influencing the extended warranty demand is $\nu_{c}{ }^{*} . \nu_{c}{ }^{*}$ is affected by the price $C_{E}$. Therefore, the proportion of items sold with warranty is determined by the price $C_{E}$. Let $C_{E 1}$ and $C_{E 2}$ be two arbitrary extended warranty prices and $C_{E 1}<C_{E 2}$. Based the assumption of customers' utility function, we can obtain the following mathematical relationships: $U_{c}\left(C_{E 1}\right)>U_{c}\left(C_{E 2}\right), E\left[U_{c}\left(\tau_{n 1} r_{c}\right)\right]>E\left[U_{c}\left(\tau_{n 2} r_{c}\right), E\left(\tau_{n 1} r_{c}\right)<\right.$ 
$E\left(\tau_{n 2} r_{c}\right), \nu_{c 1} t_{e w}<\nu_{c 2} t_{e w}, 1-G\left(\nu_{c 1}\right)>1-G\left(\nu_{c 2}\right), \sigma_{1}>\sigma_{2}$. These relationships imply that the proportion of items sold with warranty is decreasing on the extended warranty price. For the producer, the incomes mainly derive from the extended warranty incomes $C_{E} \sigma n$ and the repaired incomes on the failure product with non-extended warranty $h \tau_{n} r_{c}$. The corresponding cost is $\tau_{w} r_{p}$ and $h \tau_{n} r_{p}$.

The profit function is

$$
\Pi=C_{E} \sigma n-\tau_{w} r_{p}+\left(h \tau_{n} r_{c}-h \tau_{n} r_{p}\right)=C_{E} \sigma n-\tau_{w} r_{p}+h \tau_{n}\left(r_{c}-r_{p}\right)
$$

For notational convenience, let $d_{1}=r_{c}-r_{p}$, so Equation (3) can be expressed as

$$
\Pi=C_{E} \sigma n-\tau_{w} r_{p}+d_{1} h \tau_{n}=C_{E} \sigma n+\left(d_{1} h \tau_{n}-\tau_{w} r_{p}\right)
$$

As we can see from Equation (4), the profit is divided into the certain profit and the uncertain profit. The certain profit is the extended warranty price. The uncertain profit mainly includes maintenance income during non-warranty scope minus maintenance cost during the extended warranty period. As $C_{E} \sigma n$ exists the single optimal value, the key point is the uncertainty profit function $d_{1} h \tau_{n}-\tau_{w} r_{p}$. And let $y=d_{1} h \tau_{n}-\tau_{w} r_{p}$.

The extended warranty price is depended on the number of product failures $\tau_{w}$ and $\tau_{n}$. The number of product failures is dependent on the producer's anticipated failures rate. The failure rate during the warranty period is distributed as Gamma distribution $\psi_{p} \sim g_{p}\left(\nu_{p} \mid \alpha_{p}, \beta_{p}\right)$. $\alpha_{p}$ and $\beta_{p}$ are the shape parameter and scale parameter. The joint probability density function of $\tau_{w}$ and $\tau_{n}$ is

$$
p\left(\tau_{w}, \tau_{n}\right)=\int_{0}^{\infty} p\left(\tau_{w}\right) p\left(\tau_{n}\right) g_{p}\left(v_{p}\right) \mathrm{d} v_{p}
$$

where $\left(\tau_{w}\right)=\sigma n_{1} \nu_{p} t_{e w}, E\left(\tau_{n}\right)=(1-\sigma) n_{1} \nu_{p} t_{e w}$.

Let $C_{E 1}$ and $C_{E 2}$ be two arbitrary extended warranty prices $C_{E 1}<C_{E 2}$. Based the assumption of the producer's utility function, we can obtain the following mathematical relationships: $\sigma_{1}>\sigma_{2}, \sigma_{1} n_{1} \nu_{p} t_{e w}>\sigma_{2} n_{1} \nu_{p} t_{e w},\left(1-\sigma_{1}\right) n_{1} \nu_{p} t_{e w}\left\langle\left(1-\sigma_{2}\right) n_{1} \nu_{p}, t_{e w} E\left(\tau_{w 1}\right)\right\rangle E\left(\tau_{w 2}\right) E\left(\tau_{n 1}\right)<$ $E\left(\tau_{n 2}\right), E\left(r_{p} \tau_{w 1}\right)>E\left(r_{p} \tau_{w 2}\right), E\left(d_{1} h \tau_{n 1}\right)<E\left(d_{1} h \tau_{n 2}\right), E\left(d_{1} h \tau_{n 1}\right)-E\left(r_{p} \tau_{w 1}\right)<E\left(d_{1} h \tau_{n 2}\right)-$ $E\left(r_{p} \tau_{w 2}\right), E\left[U_{p}\left(d_{1} h \tau_{n 1}-r_{p} \tau_{w 1}\right)\right]<\left[U_{p}\left(d_{1} h \tau_{n 2}-r_{p} \tau_{w 2}\right)\right], U_{p} y_{1}<U_{p} y_{2}, y_{1}<y_{2}$. The proportion of items sold with warranty is dreasing on the extended warranty price, $\frac{\mathrm{d} \sigma}{\mathrm{d} C_{E}}<0 . y$ is increasing on the extended warranty price, $\frac{\mathrm{d} y}{\mathrm{~d} C_{E}}>0$. So

$$
\begin{gathered}
\lim _{C_{E} \rightarrow 0} \frac{\mathrm{d} \Pi}{\mathrm{d} C_{E}}=\lim _{C_{E} \rightarrow 0}\left[n_{1}\left(\sigma+\frac{\mathrm{d} \sigma}{\mathrm{d} C_{E}} C_{E}\right)+\frac{\mathrm{d} y}{\mathrm{~d} C_{E}}\right]>0, \\
\lim _{C_{E} \rightarrow \infty} \frac{\mathrm{d} \Pi}{\mathrm{d} C_{E}}=\lim _{C_{E} \rightarrow \infty}\left[n_{1}\left(\sigma+\frac{\mathrm{d} \sigma}{\mathrm{d} C_{E}} C_{E}\right)+\frac{\mathrm{d} y}{\mathrm{~d} C_{E}}\right]<0 .
\end{gathered}
$$

We can see from the above proof that the optimal extended warranty price is existence and uniqueness in the interval $(0, \infty)$.

\subsection{The Optimal Pricing Model of the Extended Warranty}

The extended warranty is the normal consumer goods, that is to say, the consumers' purchase intention is decreasing with the increasing of the extended warranty price. The optimal extended warranty price exists only achieving the profit maximization for the producer. The 
optimal extended warranty price model is constructed embodying the specific utility function and the failure rate function.

The producer' and consumers' utility function are represented by the exponential function. The corresponding utility functions are respectively $U_{p} Y=-\mathrm{e}^{-a_{1} Y}$ and $U_{c} Y=-\mathrm{e}^{b_{1} Y} \cdot a_{1} \geq$ $0, b_{1} \geq 0 . a_{1}$ is the producer's risk preference; $b_{1}$ is the consumers' risk preference; $a=0, b=0$ implies that the producer and the consumers' risk preferences are risk neutral. When $a$ and $b$ are increasing, the producer and consumers' risk preferences are higher.

$$
E\left[U_{p}(y)\right]=\sum_{\tau_{w}=0}^{\infty} \sum_{\tau_{n}=0}^{\infty}-\mathrm{e}^{-a_{1} y} p\left(\tau_{w}, \tau_{n}\right)
$$

Combing Equation (5), Equation (6) can be expressed as follows:

$$
E\left[U_{p}(y)\right]=\int_{0}^{\infty} \sum_{\tau_{w}=0}^{\infty} \sum_{\tau_{n}=0}^{\infty}-\mathrm{e}^{-a y} p\left(\tau_{w}\right) p\left(\tau_{n}\right) g_{p}\left(\nu_{p} \mid \alpha_{p}, \beta_{p}\right) \mathrm{d} \nu_{p}
$$

As $y=d k \tau_{n}-\tau_{w} r_{p}$, so Equation (7) is reduced as

$$
\begin{aligned}
E\left[U_{p}(y)\right] & =\int_{0}^{\infty} \sum_{\tau_{w}=0}^{\infty} \sum_{\tau_{n}=0}^{\infty}-\mathrm{e}^{-a_{1}\left(\mathrm{~d} h \tau_{n}-\tau_{w} r_{p}\right)} p\left(\tau_{w}\right) p\left(\tau_{n}\right) g_{p}\left(v_{p} \mid \alpha_{p}, \beta_{p}\right) \mathrm{d} v_{p} \\
& =-\int_{0}^{\infty} \sum_{\tau_{w}=0}^{\infty} \sum_{\tau_{n}=0}^{\infty} \exp \left(a_{1} \tau_{w} r_{p}-a_{1} d_{1} h \tau_{2}\right) p\left(\tau_{w}\right) p\left(\tau_{n}\right) g_{p}\left(v_{p} \mid \alpha_{p}, \beta_{p}\right) \mathrm{d} v_{p} \\
& =-E\left\{\exp \left(-v_{p}\left[(1-\sigma) n_{1} t_{e w}\left(1-\mathrm{e}^{-a_{1} d_{1} h}\right)+\sigma n_{1} t_{e w}\left(1-\mathrm{e}^{a_{1} r_{p}}\right)\right]\right) \mid \alpha_{p}, \beta_{p}\right\} \\
& \left.=-\left\{1+\beta_{p}\left[(1-\sigma) n_{1} t_{e w}\left(1-\mathrm{e}^{-a_{1} d_{1} h}\right)+\sigma n_{1} t_{e w}\left(1-\mathrm{e}^{a_{1} r_{p}}\right)\right]\right)\right\}^{-\alpha_{p}}
\end{aligned}
$$

From Equation (8), we can see

$$
y=\left(\frac{\alpha_{p}}{a_{1}}\right) \ln \left\{1+\beta_{p} n_{1} t_{e w}\left[(1-\sigma)\left(1-\mathrm{e}^{-a_{1} d_{1} h}\right)+\sigma\left(1-\mathrm{e}^{a_{1} r_{p}}\right)\right]\right\}
$$

Combing Equation (9), Equation (4) becomes

$$
\begin{aligned}
\Pi & =C_{E} \sigma n_{1}+\left(d_{1} h \tau_{n}-\tau_{w} r_{p}\right) \\
& =C_{E} \sigma n_{1}+\left(\frac{\alpha_{p}}{a_{1}}\right) \ln \left\{1+\beta_{p} n_{1} t_{e w}\left[(1-\sigma)\left(1-\mathrm{e}^{-a_{1} d_{1} h}\right)+\sigma\left(1-\mathrm{e}^{a_{1} r_{p}}\right)\right]\right\}
\end{aligned}
$$

The producer determines the optimal extended warranty price such that the expected profit is maximized. We take the partial derivation of profit with respect to the extended price in Equation (10), the result is computed as

$$
E\left[U_{p}(y)\right]=\sum_{\tau_{w}=0}^{\infty} \sum_{\tau_{n}=0}^{\infty}-\mathrm{e}^{-a_{1} y} p\left(\tau_{w}, \tau_{n}\right)
$$

In order to calculate $\frac{\mathrm{d} \sigma}{\mathrm{d} p}$, we crystallize the consumers' risk preference function $U_{c}(y)=$ $-\mathrm{e}^{b_{1} y}$, incorporating (1), we can obtain

$$
E\left[U_{c}\left(C_{E}\right)\right]=-E\left[\mathrm{e}^{b_{1} r_{c} \tau_{n}} \mid \mu=\nu_{c} t_{e w}\right]=-\exp \left[\nu_{c} t_{e w}\left(\mathrm{e}^{b_{1} r_{c}}-1\right)\right]
$$

We can see from Equation (12),

$$
\nu_{c} t_{e w}\left(\mathrm{e}^{b_{1} r_{c}}-1\right)=b_{1} C_{E}, \quad \nu_{c}=\frac{b_{1} C_{E}}{t_{e w}\left(\mathrm{e}^{b r_{c}}-1\right)},
$$




$$
\frac{d_{\omega}}{\mathrm{d} C_{E}}=\frac{\mathrm{d} \int_{v_{c}}^{\infty} g_{c}\left(v_{c} \mid \alpha_{c}, \beta_{c}\right) \mathrm{d} v_{c}}{\mathrm{~d} C_{E}} \frac{\mathrm{d} v_{c}}{\mathrm{~d} C_{E}}=g_{c}\left(v_{c} \mid \alpha_{c}, \beta_{c}\right) \frac{b_{1}}{t_{e w}\left(\mathrm{e}^{b_{1} r_{c}}-1\right)}
$$

Integrating Equation (13), Equation (11) are as shown below:

$$
\begin{aligned}
& \frac{\partial \prod}{\partial C_{E}} \\
= & n_{1}\left[\int_{v_{c}}^{\infty} g_{c}\left(v_{c} \mid \alpha_{c}, \beta_{c}\right) \mathrm{d} v_{c}+g_{c}\left(v_{c} \mid \alpha_{c}, \beta_{c}\right) \frac{b_{1} C_{E}}{t_{e w}\left(\mathrm{e}^{b_{1} r_{c}}-1\right)}\right] \\
& +\left(\frac{\alpha_{p}}{a_{1}}\right) \frac{\beta_{p} n_{1} t_{e w} g_{c}\left(v_{c} \mid \alpha_{c}, \beta_{c}\right) \frac{b_{1}}{t_{e w}\left(\mathrm{e}^{\left.b_{1} r_{C}-1\right)}\right.}\left(\mathrm{e}^{-a_{1} d_{1} h}-\mathrm{e}^{a_{1} r_{p}}\right)}{1+\beta_{p} n_{1} t_{e w}\left[\left(1-\int_{v_{c}}^{\infty} g_{c}\left(v_{c} \mid \alpha_{c}, \beta_{c}\right) \mathrm{d} v_{c}\right)\left(1-\mathrm{e}^{-a_{1} d_{1} h}\right)+\left(\int_{v_{c}}^{\infty} g_{c}\left(v_{c} \mid \alpha_{c}, \beta_{c}\right) \mathrm{d} v_{c}\right)\left(1-\mathrm{e}^{a_{1} r_{p}}\right)\right]} \\
= & 0
\end{aligned}
$$

It directly follows from Equation (14) that

$$
\begin{aligned}
= & C_{E}^{*}-\int_{v_{c}}^{\infty} g_{c}\left(v_{c} \mid \alpha_{c}, \beta_{c}\right) \mathrm{d} v_{c} \\
& \left.+\left(\frac{\alpha_{p}}{a_{1}}\right) \frac{\beta_{p} t g_{c}\left(v_{c} \mid \alpha_{c}, \beta_{c}\right) \frac{b_{1}}{t_{e w}\left(\mathrm{e}^{\left.b_{1} r_{C-1}\right)}\right.}\left(\mathrm{e}^{-a_{1} d_{1} h}-\mathrm{e}^{a_{1} r_{p}}\right)}{1-\beta_{p} n_{1} t_{e w}\left[\left(1-\int_{v_{c}}^{\infty} g_{c}\left(v_{c} \mid \alpha_{c}, \beta_{c}\right) \mathrm{d} v_{c}\right)\left(1-\mathrm{e}^{-a_{1} d_{1} h}\right)+\int_{v_{c}}^{\infty} g_{c}\left(v_{c} \mid \alpha_{c}, \beta_{c}\right) \mathrm{d} v_{c}\left(1-\mathrm{e}^{a_{1} r_{p}}\right)\right]}\right\} \\
& \cdot \frac{t_{e w}\left(\mathrm{e}^{b_{1} r_{c}}-1\right)}{g_{c}\left(v_{c} \mid \alpha_{c}, \beta_{c}\right) b_{1}}
\end{aligned}
$$

\section{A Numerical Example}

The optimal extended warranty price model tells us that the critical influences on the optimal extended warranty price mainly include: The producer' and consumers' risk preferences; the producer and consumers' per-occasion maintenance cost; the incompatible degree of maintenance technology for the producer and the extended warranty period. We adopt the simulation method to study the influence relation of the main factors on the optimal extended warranty price.

a) Effect of the risk preference

The optimal extended warranty price increases as the producer's risk preference parameter is increased. Figure 1 shows that the effect of the producer's risk preference to the optimal extended warranty price. This implies that, as the producer becomes more risk-averse, the producer will impose a higher extended warranty price. As the consumers' risk preference parameter is increased, the optimal extended warranty price decreases. The effect of the consumers' risk preference to the optimal extended warranty price is demonstrated in Figure 2. It demonstrated that as the consumers become more risk-preference, the consumers will pay a higher extended warranty price. The parameter values, other specified, are set as following: $n_{1}=2, h=0.5, r_{c}=2, r_{p}=1, d=1, \alpha_{c}=2, \beta_{c}=2, t=1, \nu_{c}=2, \alpha_{p}=2, \beta_{p}=2$. We compare the influencing degree between the producer's risk-preference and consumers' risk-preference on the optimal extended warranty price and draw the conclusion: The influence degree of the consumers' risk preference to the optimal extended warranty price is greater than the influence degree of the producer's risk preference to the optimal extended warranty price. Since the 
consumers' risk preference is decreased more significantly than the producer's risk preference is increased, the consumers' risk preference has more impact on the optimal extended warranty price than the producer's risk preference.

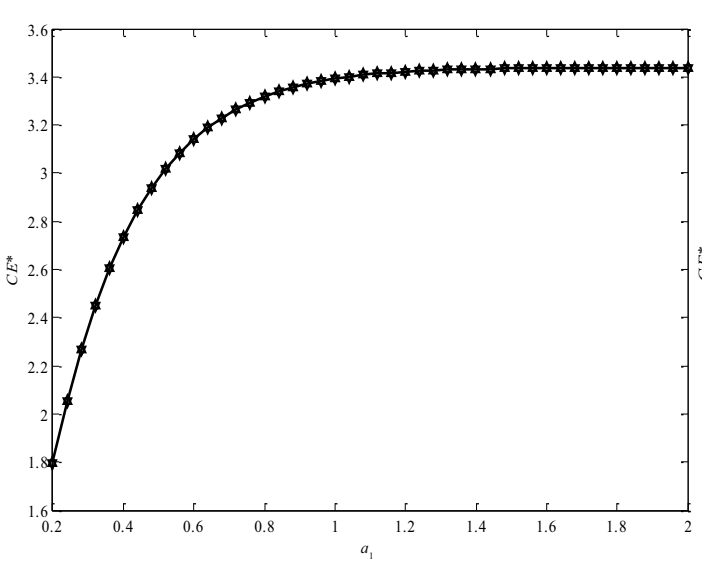

Figure 1 Effect of the producer's risk preference on the optimal extended warranty price

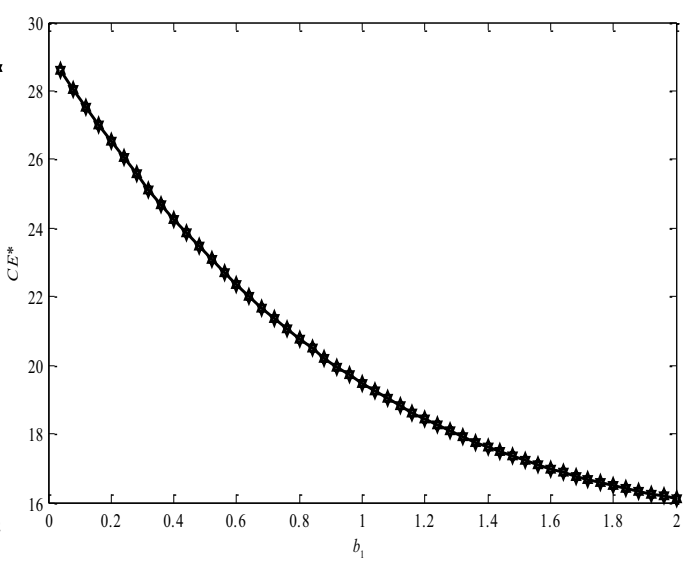

Figure 2 Effect of the consumers' risk preference on the optimal extended warranty price

b) Effect of the incompatible degree of maintenance technology

To analyze the effect of the incompatible degree of maintenance technology on the optimal extended warranty price, we vary $h$ from 0 to 1 by 0.02 . $h=0$ implies that the non-warranted items don't return the original producer for maintenance due to malfunctions, whereas $h=1$ means that non-warranted items all return the original producer for maintenance when the fault appears. Figure 3 shows the effect of the incompatible degree of maintenance technology on the optimal extended warranty price. In Figure 3, the optimal extended warranty price increases as the incompatible degree of maintenance technology become much greater. The increasing degrees of the optimal extended warranty price approximately keep stable.

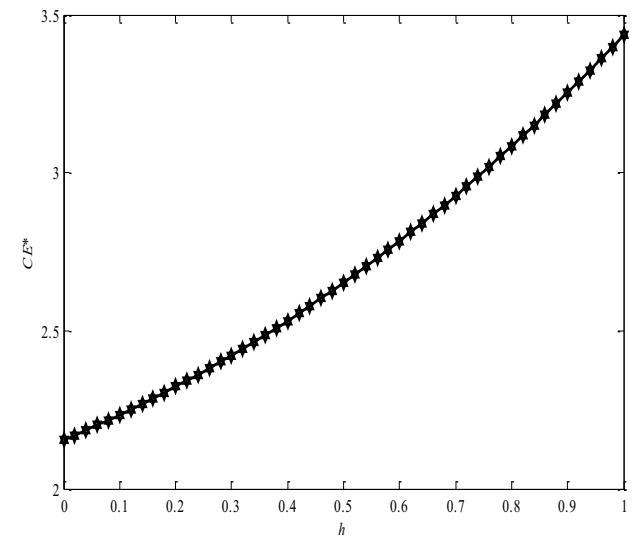

Figure 3 Effect of the incompatible degree of maintenance technology on the optimal extended warranty price

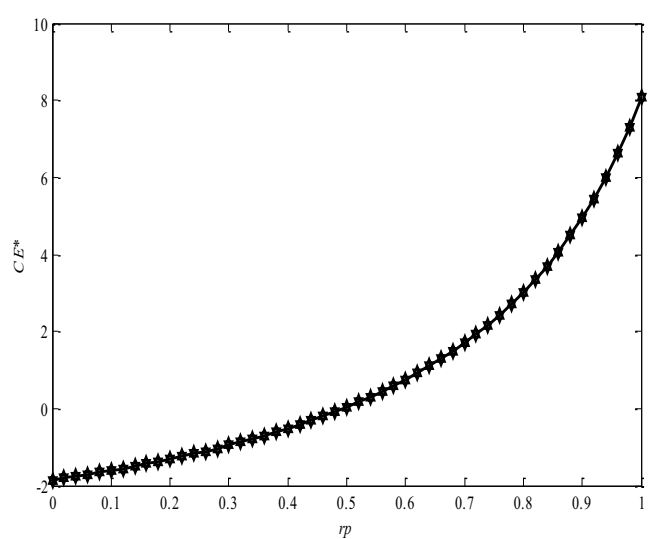

Figure 4 Effect of the producer's peroccasion maintenance cost on the optimal extended warranty price 
c) Effect of the per-occasion maintenance cost

As the value of the producer's per-occasion maintenance cost distribution is increased from 0 to 1 with the variance fixed at 0.02 , the optimal extended warranty price rises from 1.6 to 2.4 in Figure 4. Figure 4 implies that, the extended warranty price curve is consistently higher as the producer's per-occasion maintenance cost to be higher. As the value of the consumers' per-occasion maintenance cost distribution is increased from 0 to 1 with the variance fixed at 0.02, the optimal extended warranty price rises from 0 to 10 in Figure 5. Figure 5 implies that, the extended warranty price curve is consistently higher as the consumers' per-occasion maintenance cost to be higher. We can draw the difference between the influence degrees of the producer's per-occasion maintenance cost and the consumers' per-occasion maintenance cost to the optimal extended warranty price. The influence degree of the consumers' per-occasion maintenance cost to the optimal extended warranty price is greater than the influence degree of the producer's per-occasion maintenance cost to the optimal extended warranty price.

d) Effect of the extended warranty period

As expected, the optimal extended warranty price increases approximately linearly from 10.45 to 27.85 as the extended warranty period is increased from 1 to 2 in Figure 6 . The parameter values, other specified, are set as following: $n_{1}=2, h=0.5, r_{c}=2, r_{p}=1, d=$ $1, \alpha_{c}=2, \beta_{c}=2, a_{1}=0.5, b_{1}=0.5, \nu_{c}=2, \alpha_{p}=2, \beta_{p}=2$. It indicated that, as the extended warranty period is increased, the producer will establish the higher extended warranty price.

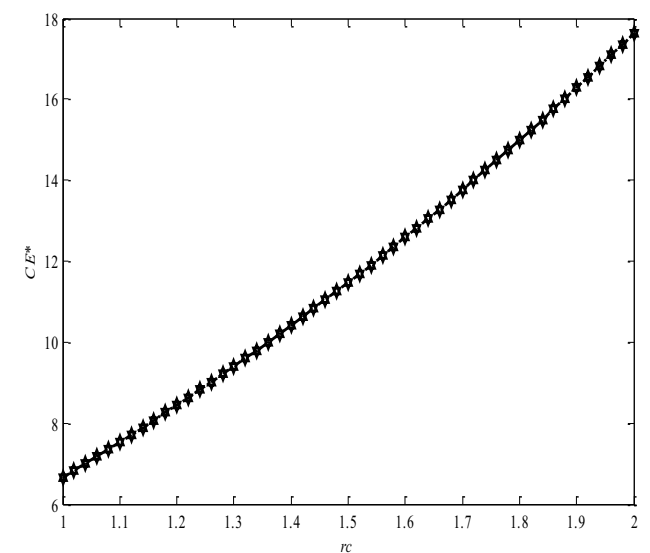

Figure 5 Effect of the consumers' peroccasion maintenance cost on the optimal extended warranty price

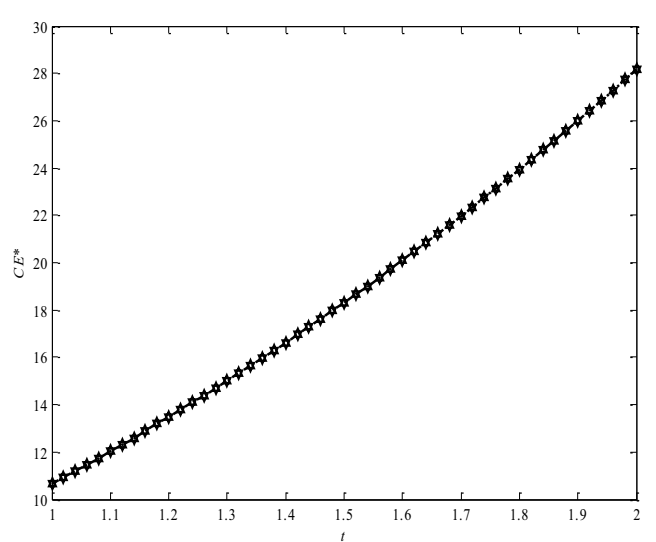

Figure 6 Effect of the extended warranty period on the optimal extended warranty price

\section{Conclusion}

The paper studies the mechanism of indirect network effect and designs the optimal extended warranty price with indirect network effect. The paper demonstrates the following conclusions: First, the producer's risk preference, the producer and consumers' per-occasion maintenance cost, and the incompatible degree of the producer's maintenance technology as well as the extended warranty period all positively influence the optimal extended warranty price; while the consumers' risk preference has the negative impact on the optimal extended warranty price. Second, the influence degree of the consumers' risk preference and per-occasion maintenance 
cost to the extended warranty price is greater than that of the producer's risk preference and per-occasion maintenance cost separately.

\section{References}

[1] Katz M L, Shapiro C. Network externalities, competition and compatibility. The American Economic Review, 1985, 75(3): 424-440.

[2] Berke T M, Zaino N A. Warranties: What are they? What do they really cost? Proceedings of the 1991 IEEE Annual Reliability and Maintainability, Symposium, 1991: 326-330.

[3] Lutz N A, Padmanabhan V. Why do we observe minimal warranties? Marketing Science, 1995, 14: 417-441.

[4] Chun Y H, Tang K. Determining the optimal warranty price based on the producer's and customers' risk preferences. European Journal of Operational Research, 1995, 85: 97-110.

[5] Padmanabhan V. Usage heterogeneity and extended warranties. Journal of Economics and Management Strategy, 1995, 4: 33-53.

[6] Spence A M. Consumer misperception product failure and product liability. Review of Economic Studies, 1977(44): 561-572.

[7] Lutz N A. Warranties as signals under consumer moral hazard. The RAND Journal of Economics, 1989, 20(2): 239-255.

[8] Heal G. Guarantees and risk-sharing. Review of Economic Studies, 1977, 44(3): 549-560.

[9] Akerlof G A. The market for "lemons": Quality uncertainty and the market mechanism. The Quarterly Journal of Economics, 1970, 84(3): 488-500.

[10] Padmanabhan V, Rao R C. Warranty policy and extended service contracts: Theory and an application to automobiles. Marketing Science, 1993, 12(3): 230-247.

[11] Chen Z Q, Ross T W. Why are extended warranties so expensive? Economics Letters, 1994, 45: 253-257.

[12] Ladany S P, Shore H. Profit maximizing warranty period with sales expressed by a demand function. Quality and Reliability Engineering International, 2007, 23(3): 291-293.

[13] Chen J A, Chien Y H. Renewing warranty and preventive maintenance for product with failure penalty post-warranty. Quality and Reliability Engineering International, 2007, 23: 107-121.

[14] Murthy D N P, Blischke W R. Strategic warranty management: A life-cycle approach. IEEE Transactions on Engineering Management, 2000, 47(1): 40-54.

[15] Price L J, Dawar N. The joint effects of brands and warranties in signaling new product quality. Journal of Economic Psychology, 2002, 23(2): 165-190.

[16] Lawless J F, Crowder M J, Lee K A. Monitoring warranty claims with consums. Technimetrics, 2012, 54(3): 269-278.

[17] Zhou C W, Chinnam R B, Korostelev A. Hazard rate models for early detection of reliability problems using information from warranty databases and upstream supply chain. International Journal of Production Economics, 2012, 139(1): 180-195

[18] Wu S M. Warranty claim analysis considering human factors. Reliability Engineering \& System Safety, 2008, 96(1): 131-138.

[19] Lawless J F, Crowder M J, Lee K A. Analysis of reliability and warranty claims in product with age and usage scales. Technimetrics, 2009, 51(1): 14-24.

[20] Ye Z S, Murthy D N P, Xie M, et al. Optimal burn-in for repairable product sold with a two-dimensional warranty. IIE Transactions, 2013, 45(2): 164-176. 